\title{
Scintillation correlation between forward and return spherical waves
}

\author{
Nicolas Perlot* and Dirk Giggenbach \\ Institute of Communications and Navigation, Deutsches Zentrum für Luft- und Raumfahrt (DLR) \\ Oberpfaffenhofen, D-82234 Wessling, Germany \\ ${ }^{*}$ Corresponding author: nicolas.perlot@dlr.de
}

Received 3 October 2011; revised 12 January 2012; accepted 12 January 2012;

posted 12 January 2012 (Doc. ID 155945); published 16 May 2012

\begin{abstract}
According to the point-source point-receiver (PSPR) reciprocity, the received field remains equal when the positions of a point source and point receiver are interchanged. We extend the PSPR scenario to a finite receiver that spatially averages scintillation over its aperture. By use of weak-fluctuation theory, an analytical expression for the correlation coefficient between the received powers at both link ends is provided. The effects of turbulence profile, receiver aperture size, and central obscuration on the correlation are assessed. Because correlation is obtained to the detriment of antenna gain and aperture averaging, the net benefit of the channel reciprocity is highly scenario dependent. () 2012 Optical Society of America

OCIS codes: $\quad 010.1330,030.1670,060.2605$
\end{abstract}

\section{Introduction}

Four decades ago, it was shown theoretically that the propagation of a monochromatic spherical wave has a reciprocity property in a refractive medium: the received field remains equal when the positions of the point source and point receiver are interchanged $[1,2]$. Recent experiments on bidirectional optical links through the atmosphere have demonstrated the correlation between the oppositely directed signals $[\underline{3}, \underline{4}]$.

The relation between two waves propagating through a common turbulent volume has been intensively investigated by the lidar community [5-7]. When a laser pulse is reflected by a target and measured near the laser source, a "backscatter amplification effect" is observed as a result of correlated turbulence-induced perturbations of the "incident" and "reflected" waves. However, theoretical results from the lidar community cannot be directly applied to a bidirectional link. Instead of emitting a single wave that propagates twice through the same turb-

$1559-128 \mathrm{X} / 12 / 152888-06 \$ 15.00 / 0$

(C) 2012 Optical Society of America ulent volume, a bidirectional link consists of two distinct waves emitted and received by distinct transceivers and propagating in opposite directions. The lidar signal is generally analyzed in a single plane (i.e., the plane of emission and reception), whereas a bidirectional link provides two signals analyzed in two different planes. The type of bidirectional link under consideration is depicted in Fig. 1. We use the terms "forward" and "return" link, which should not be confused with the "incident" and "reflected" lidar waves. We assume that the forwardwave transmitter (forward Tx) is located in the center of the return-wave receiver $(\mathrm{Rx})$, and vice versa at the other link end. Thus, both links have the same axis. Also shown is a thin transverse perturbation screen $\Psi$ at a distance $z_{1}$ from the forward source.

Our purpose is to quantify this correlation between the two signals for different Rx aperture sizes and different turbulence paths. The paper is organized as follows. In Section 2, based on the HuygensFresnel principle, the intensity field at one link end is expressed as a function of the intensity field at the other end when a single perturbation screen lies on the path. Based on the Rytov theory, a general expression for the correlation coefficient is given in 


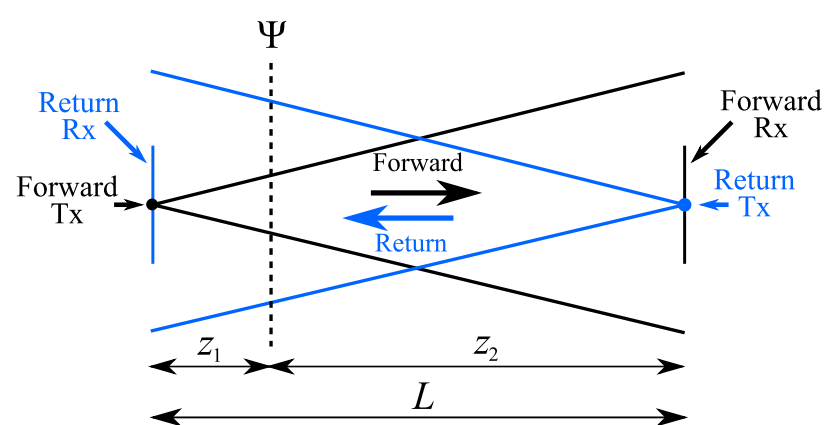

Fig. 1. (Color online) Propagation of a spherical wave in the forward and return directions. The turbulent screen $\Psi$ is shown at a distance $z_{1}$ from the point source, which is transmitting in the forward direction.

Section 3. Whereas Section 4 considers the case of a single PS on the path, Section $\underline{5}$ deals with paths of constant turbulence strength and vertical paths. We then conclude and mention some prospective activities.

\section{Huygens-Fresnel Principle}

We consider a single perturbation screen (PS) on the path as depicted in Fig. 1. By means of the HuygensFresnel principle, we express the received forward field $U_{F}(\rho)$ as a function of the forward field $U_{F, 0}(\mathbf{s})$ at the PS [8]:

$$
\begin{aligned}
U_{F}(\boldsymbol{\rho})= & \frac{\exp \left(j k z_{2}\right)}{j \lambda z_{2}} \exp \left(j \frac{k \rho^{2}}{2 z_{2}}\right) \int U_{F, 0}(\mathbf{s}) \\
& \times \exp \left(j \frac{k s^{2}}{2 z_{2}}\right) \exp \left(j \frac{k}{z_{2}} \mathbf{s} \cdot \boldsymbol{\rho}\right) \mathrm{d} \mathbf{s}
\end{aligned}
$$

$\rho$ and $\mathbf{s}$ are respectively the Rx-plane and the screenplane (two-dimensional) vectors. The expression for the forward spherical wave at the PS is

$$
U_{F, 0}(\mathbf{s})=\frac{A_{F}}{z_{1}} \exp \left[j k\left(\frac{s^{2}}{2 z_{1}}+z_{1}\right)\right] \Psi(\mathbf{s}),
$$

where the PS transmission function $\Psi(\mathbf{s})$ takes arbitrary complex values and $A_{F}$ is the constant wave amplitude factor. To obtain the return fields $U_{R}$ and $U_{R, 0}$ in the Rx plane and screen plane, respectively, we simply interchange $z_{1}$ and $z_{2}$ and replace the amplitude factor $A_{F}$ by $A_{R}$. Because our goal parameter, i.e., the correlation coefficient, is independent of the mean values of the assessed variables, we assume $A_{F}=A_{R}$ without loss of generality. The relation between the forward and return waves can be written as

$$
U_{R}(\boldsymbol{\rho})=U_{F}\left(\frac{z_{2}}{z_{1}} \boldsymbol{\rho}\right) \exp \left[j \frac{k \rho^{2}}{2 z_{1}^{2}}\left(z_{1}-z_{2}\right)\right]
$$

from which we deduce the relation between the received intensities $I_{F}$ and $I_{R}$ :

$$
I_{R}(\rho)=I_{F}\left(\frac{z_{2}}{z_{1}} \rho\right)
$$

Thus, the intensities in the forward and return $\mathrm{Rx}$ planes are the same but with different spatial scales according to the position of the perturbation screen.

\section{Correlation Coefficient for a Finite Aperture}

The covariance $\sigma_{F R}^{2}$ between the forward and return Rx powers, $P_{F}$ and $P_{R}$, is given by

$$
\begin{aligned}
\sigma_{F R}^{2}= & \left\langle P_{F} P_{R}\right\rangle-\left\langle P_{F}\right\rangle\left\langle P_{R}\right\rangle \\
= & \left\langle\int I_{F}\left(\rho_{1}\right) W_{F}\left(\rho_{1}\right) \mathrm{d} \rho_{1} \int I_{R}\left(\rho_{2}\right) W_{R}\left(\rho_{2}\right) \mathrm{d} \rho_{2}\right\rangle \\
& -\left\langle\int I_{F}\left(\rho_{1}\right) W_{F}\left(\rho_{1}\right) \mathrm{d} \rho_{1}\right\rangle\left\langle\int I_{R}\left(\rho_{2}\right) W_{R}\left(\rho_{2}\right) \mathrm{d} \rho_{2}\right\rangle .
\end{aligned}
$$

The angle brackets denote ensemble averaging. The aperture window function of the forward receiver is defined by

$$
W_{F}(\tilde{\mathbf{n}})= \begin{cases}1, & \text { for } \tilde{\mathbf{n}} \text { inside aperture } \\ 0, & \text { otherwise }\end{cases}
$$

$W_{R}(\rho)$ is defined similarly. Assuming $\left\langle I_{F}\right\rangle=\left\langle I_{R}\right\rangle=1$ and merging the two integrals in Eq. (ㅁ), we write

$$
\left.\sigma_{F R}^{2}=\iint I_{F}\left(\tilde{\mathbf{n}}_{1}\right) I_{R}\left(\tilde{\mathbf{n}}_{2}\right)-1\right\rangle W_{F}\left(\tilde{\mathbf{n}}_{1}\right) W_{R}\left(\tilde{\mathbf{n}}_{2}\right) \mathrm{d} \tilde{\mathbf{n}}_{1} \mathrm{~d} \tilde{\mathbf{n}}_{2} .
$$

We now assume weak scintillation and apply the Rytov propagation theory. This assumption allows us to consider the received intensity field as the sum of independent perturbations caused by turbulence along the propagation path. For the forward link, we thus write

$$
I_{F}(\rho)=\int_{0}^{L} i_{F}\left(\rho ; z_{1}\right) \mathrm{d} z_{1},
$$

where $i_{F}\left(\rho ; z_{1}\right)$ is the contribution to the received intensity field made by the thin turbulence screen, located at a distance $z_{1}$ from the forward transmitter. Furthermore, we describe $B_{F}(r)$, the spatial covariance function of the forward Rx intensity. Because the $\mathrm{Rx}$ intensity fields are assumed to be isotropic and homogeneous stochastic fields, the covariance between two points of these fields depends only on their separation distance $r$. Still relying on the Rytov theory, $B_{F}(r)$ is written as the sum of independent contributions cumulated along the propagation path:

$$
B_{F}(r)=\int_{0}^{L} b_{F}\left(r ; z_{1}\right) \mathrm{d} z_{1} .
$$

The covariance contribution $b_{F}\left(r ; z_{1}\right)$ for a spherical wave is given by [7] 


$$
\begin{aligned}
b_{F}(r ; z)= & 0.033 \times 8 \pi^{2} k^{2} C_{n}^{2}\left(z_{1}\right) \int_{0}^{\infty} \kappa^{-8 / 3} J_{0}\left(\kappa r \frac{z_{1}}{L}\right) \\
& \times\left(1-\cos \frac{\kappa^{2} z_{1} z_{2}}{k L}\right) \mathrm{d} \kappa
\end{aligned}
$$

with $C_{n}^{2}\left(z_{1}\right)$ being the constant of the turbulence structure function at a distance $z_{1}$ and $J_{0}$ the Bessel function of the first kind and order zero. Now, using Eq. (8), the intensity covariance term in Eq. (7) can be written as

$$
\begin{aligned}
\left\langle I_{F}\left(\tilde{\mathbf{n}}_{1}\right) I_{R}\left(\tilde{\mathbf{n}}_{2}\right)\right\rangle-1= & \left\langle\int_{0}^{L} i_{F}\left(\tilde{\mathbf{n}}_{1} ; z_{1}\right) \mathrm{d} z_{1} \int_{0}^{L} i_{R}\left(\tilde{\mathbf{n}}_{2} ; z_{1}\right) \mathrm{d} z_{1}\right\rangle \\
& -\left(\int_{0}^{L}\left\langle i_{F}\right\rangle \mathrm{d} z_{1}\right)^{2} \\
= & \int_{0}^{L} \int_{0}^{L}\left\langle i_{F}\left(\tilde{\mathbf{n}}_{1} ; z_{1}^{*}\right) i_{R}\left(\tilde{\mathbf{n}}_{2} ; z_{1}\right)\right. \\
& \left.-\left\langle i_{F}\right\rangle^{2}\right\rangle \mathrm{d} z_{1}^{*} \mathrm{~d} z_{1}
\end{aligned}
$$

where in the first step we used $1=\int_{0}^{L}\left\langle i_{F}\right\rangle \mathrm{d} z_{1}$. Introducing Eq. ()ㅡ) into Eq. (11) yields

$$
\begin{aligned}
\left\langle I_{F}\left(\tilde{\mathbf{n}}_{1}\right) I_{R}\left(\tilde{\mathbf{n}}_{2}\right)\right\rangle-1= & \int_{0}^{L} \int_{0}^{L}\left\langle i_{F}\left(\tilde{\mathbf{n}}_{1} ; z_{1}^{*}\right) i_{F}\left(\frac{z_{2}}{z_{1}} \tilde{\mathbf{n}}_{2} ; z_{1}\right)\right. \\
& \left.\times-\left\langle i_{F}\right\rangle^{2}\right\rangle \mathrm{d} z_{1}^{*} \mathrm{~d} z_{1} .
\end{aligned}
$$

The on-axis correlation distance of $i_{F}\left(z_{1}\right)$ is much smaller than both the path length $L$ and the variations of $C_{n}^{2}\left(z_{1}\right)$. It results that the covariance of $i_{F}\left(z_{1}\right)$ in Eq. (12) has some appreciable value only when the difference $\left|z_{1}^{*}-z_{1}\right|$ is close to zero. With $z_{1}^{*} \cong z_{1}$, the inner integral of Eq. (12) corresponds to the contribution $b_{F}\left(z_{1}\right)$ of turbulence at $z_{1}$ to the total intensity covariance $B_{F}$ :

$$
\begin{aligned}
& \int_{0}^{L}\left\langle i_{F}\left(\rho_{1} ; z_{1}^{*}\right) i_{F}\left(\frac{z_{2}}{z_{1}} \rho_{2} ; z_{1}\right)-\left\langle i_{F}\right\rangle^{2}\right\rangle \mathrm{d} z_{1}^{*} \\
& \quad \approx \int_{0}^{L}\left\langle i_{F}\left(\rho_{1} ; z_{1}\right) i_{F}\left(\frac{z_{2}}{z_{1}} \rho_{2} ; z_{1}\right)-\left\langle i_{F}\right\rangle^{2}\right\rangle \mathrm{d} z_{1}^{*} \\
& \quad \approx b_{F}\left(\left|\frac{z_{2}}{z_{1}} \rho_{2}-\rho_{1}\right| ; z_{1}\right) .
\end{aligned}
$$

Equation (12) thus becomes

$$
\left\langle I_{F}\left(\tilde{\mathbf{n}}_{1}\right) I_{R}\left(\tilde{\mathbf{n}}_{2}\right)\right\rangle-1 \approx \int_{0}^{L} b_{F}\left(\left|\frac{z_{2}}{z_{1}} \tilde{\mathbf{n}}_{2}-\tilde{\mathbf{n}}_{1}\right| ; z_{1}\right) \mathrm{d} z_{1} .
$$

Inserting Eq. (14) into Eq. (7) leads to

$$
\begin{aligned}
\sigma_{F R}^{2}= & \int_{\mathbb{R}^{2}} \int_{\mathbb{R}^{2}} \int_{0}^{L} b_{F}\left(\left|\frac{z_{2}}{z_{1}} \tilde{\mathbf{n}}_{2}-\tilde{\mathbf{n}}_{1}\right| ; z_{1}\right) \\
& \times W_{F}\left(\tilde{\mathbf{n}}_{1}\right) W_{R}\left(\tilde{\mathbf{n}}_{2}\right) \mathrm{d} z_{1} \mathrm{~d} \tilde{\mathbf{n}}_{1} \mathrm{~d} \tilde{\mathbf{n}}_{2} .
\end{aligned}
$$

Applying a factor $z_{1} / z_{2}$ to the integral variable $\rho_{2}$, Eq. (15) becomes

$$
\begin{aligned}
\sigma_{F R}^{2}= & \int_{0}^{L}\left(\frac{z_{1}}{z_{2}}\right)^{2} \int_{\mathbb{R}^{2}} \int_{\mathbb{R}^{2}} b_{F}\left(\mid \tilde{\mathbf{n}}_{2}\right. \\
& \left.-\tilde{\mathbf{n}}_{1} \mid ; z_{1}\right) W_{F}\left(\tilde{\mathbf{n}}_{1}\right) W_{R}\left(\frac{z_{1}}{z_{2}} \tilde{\mathbf{n}}_{2}\right) \mathrm{d} \tilde{\mathbf{n}}_{1} \mathrm{~d} \tilde{\mathbf{n}}_{2} \mathrm{~d} z_{1} .
\end{aligned}
$$

For apertures with circular symmetry, Fried has shown how the two integrals over $\mathbb{R}^{2}$ can be rewritten as a single integral over $\mathbb{R}^{+}[9]$ :

$$
\sigma_{F R}^{2}=2 \pi \int_{0}^{L} \int_{0}^{\infty}\left(z_{1} / z_{2}\right)^{2} b_{F}\left(r ; z_{1}\right) H_{F R}\left(r ; z_{1}\right) r \mathrm{~d} r \mathrm{~d} z_{1},
$$

where

$$
H_{F R}\left(r ; z_{1}\right)=\int W_{F}\left(\mathbf{r}^{\prime}\right) W_{R}\left(\frac{z_{1}}{z_{2}}\left(\mathbf{r}^{\prime}+\mathbf{r}\right)\right) \mathrm{d} \mathbf{r}^{\prime} .
$$

We can now express the correlation coefficient, defined by

$$
\mu_{F R} \equiv \sigma_{F R}^{2} / \sigma_{F} \sigma_{R},
$$

where $\sigma_{F}^{2}$ is the variance of the forward Rx power, given by

$$
\sigma_{F}^{2}=2 \pi \int_{0}^{\infty} B_{F}(r) H_{F}(r) r \mathrm{~d} r
$$

with

$$
H_{F}(r)=\int W_{F}\left(\mathbf{r}^{\prime}\right) W_{F}\left(\mathbf{r}^{\prime}+\mathbf{r}\right) \mathrm{d} \mathbf{r}^{\prime},
$$

and where the variance $\sigma_{R}^{2}$ of the return Rx power is expressed similarly, replacing $R$ with $F$ in the above two equations. Inserting Eqs. (17) and (20) into Eq. (19), we finally obtain

$$
\mu_{F R}=\frac{\int_{0}^{L}\left(z_{1} / z_{2}\right)^{2} \int_{0}^{\infty} b_{F}\left(r ; z_{1}\right) H_{F R}\left(r ; z_{1}\right) r \mathrm{~d} r \mathrm{~d} z_{1}}{\sqrt{\int_{0}^{\infty} B_{F}(r) H_{F}(r) r \mathrm{~d} r \int_{0}^{\infty} B_{R}(r) H_{R}(r) r \mathrm{~d} r}} .
$$

In arriving at Eq. (22), Rx apertures have rotational symmetry. We additionally relate both receivers according to $W_{R}(\tilde{\mathbf{n}})=W_{F}(\tilde{\mathbf{n}})(\tilde{\mathbf{n}} / m)$, where $m$ is a scaling factor. We denote by $D_{F}$ and $D_{R}$ the forward and return $\mathrm{Rx}$ diameters, which are thus related by $D_{R}=m D_{F}$, and introduce the forward $\mathrm{Rx}$ area $a_{F} \equiv \int W_{F}(\rho) \mathrm{d} \rho$. To help analyze the limit cases for $\mu_{F R}$, Table 1 provides the "height" and the "width" of the functions involved in Eq. (22). The "height" corresponds to their maximum value at $r=0$. The "width" is the $r$-value above which the function is close to zero. The correlation widths $\rho_{F}$ and $\rho_{R}$ are defined as the widths of $b_{F}\left(r ; z_{1}\right)$ and $b_{R}\left(r ; z_{1}\right)$, respectively. 
Table 1. Main Characteristics of Functions Involved in Eq. (22)

\begin{tabular}{lcc}
\hline Function & Function & Function Width \\
$f(r)$ & Height $f(r=0)$ & over $r$ \\
\hline$b_{F}\left(r ; z_{1}\right)$ & $\propto C_{n}^{2}\left(z_{1}\right)\left(z_{2} z_{1}\right)^{5 / 6}$ & $\rho_{F}=\sqrt{\lambda L z_{2} / z_{1}}$ \\
$b_{R}\left(r ; z_{1}\right)$ & $\propto C_{n}^{2}\left(z_{1}\right)\left(z_{2} z_{1}\right)^{5 / 6}$ & $\rho_{R}=\sqrt{\lambda L z_{1} / z_{2}}$ \\
$H_{F R}\left(r ; z_{1}\right)$ & Circular Rx apertures & $\frac{1}{2} D_{F}\left(1+m z_{2} / z_{1}\right)$ \\
& without obscuration: \\
& $a_{F}$ for $z_{1} / m z_{2} \leq 1$ \\
$a_{F}\left(m z_{2} / z_{1}\right)^{2}$ for \\
$H_{F}(r)$ & $z_{1} / m z_{2}>1$ \\
$H_{R}(r)$ & $a_{F}$ & $D_{F}$ \\
\hline & $m^{2} a_{F}$ & $D_{R}$ \\
\hline
\end{tabular}

\section{Single-PS Analysis}

Assuming that the perturbations consist of a single PS at position $z_{1}$, Table 2 gives the values of $\mu_{F R}$ under certain limit conditions. Figure 2 shows the coefficient $\mu_{F R}$ as a function of the PS position along the path. In the left panel, both receivers have equal geometry, with $D_{R}=D_{F}$. The case of a circular central obscuration defined by the obscuration ratio $q$ (ratio of inner diameter to outer diameter) is considered. The obstruction ratio $q$ is assumed equal for both receivers. Correlation reduction due to central obscuration is particularly strong when the central obscuration on one link side encompasses 1 correlation width more than the outer Rx diameter on the other link side. This condition can be written as $q D_{R} / \rho_{R}-D_{F} / \rho_{F} \approx 1$ or, symmetrically, $q D_{F} / \rho_{F}-D_{R} / \rho_{R} \approx 1$.

In the right panel of Fig. 2 , it can be observed how $\mathrm{Rx}$ apertures of different sizes (here $D_{R}=3 D_{F}$ is assumed) modify the symmetry of $\mu_{F R}$ with respect to the PS position. The full correlation is obtained for a PS located at $z_{1} / L=m /(m+1)$.

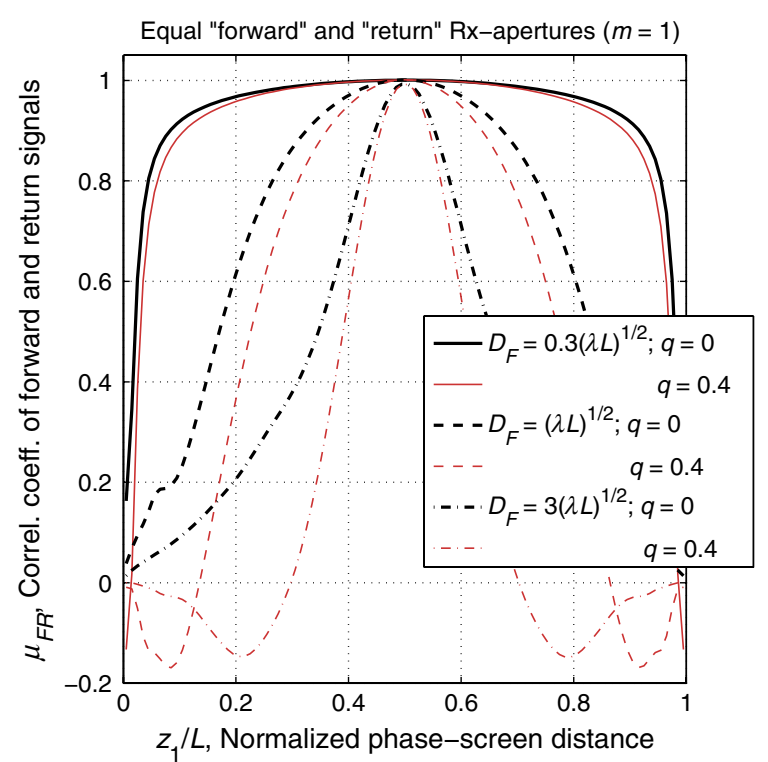

Table 2. Correlation Coefficients $\mu_{F R}$ under Limit Conditions for a Single Turbulent Screen along the Path

\begin{tabular}{|c|c|c|c|}
\hline Path Region & \multicolumn{2}{|c|}{ Limit Condition } & $\mu_{F R}$ \\
\hline$\frac{z_{1}}{m z_{2}} \leq 1$ & $\begin{array}{c}z_{1} / z_{2} \gg \lambda L / D_{F}^{2} \\
z_{1} / z_{2} \gg m^{2}\left(D_{F}^{2} / \lambda L\right)\end{array}$ & $\begin{array}{l}\text { (i.e., } D_{F} \gg \rho_{F} \text { ) } \\
\text { (i.e., } D_{R} \ll \rho_{R} \text { ) }\end{array}$ & $\begin{array}{l}\approx z_{1} / m z_{2} \\
\approx 1\end{array}$ \\
\hline$\frac{z_{1}}{m z_{2}}>1$ & $\begin{array}{c}z_{1} / z_{2} \ll \lambda L / D_{F}^{2} \\
z_{1} / z_{2} \ll m^{2}\left(D_{F}^{2} / \lambda L\right)\end{array}$ & $\begin{array}{l}\text { (i.e., } D_{F} \ll \rho_{F} \text { ) } \\
\text { (i.e., } D_{R} \gg \rho_{R} \text { ) }\end{array}$ & $\begin{array}{l}\approx 1 \\
\approx m z_{2} / z_{1}\end{array}$ \\
\hline
\end{tabular}

\section{Continuous- $C_{n}^{2}$ Paths}

In Fig. $\underline{3}$, the correlation coefficient is shown as a function of $D_{F} / \sqrt{\lambda L}$, considering a horizontal path characterized by a constant $C_{n}^{2}$. A symmetric link is considered with $m=1$ (i.e., $D_{R}=D_{F}$ ), as well as an asymmetrical link with $m=3$. Although correlation decreases as $D_{F}$ increases, we note that the correlation does not tend to zero. This persistent correlation is caused by turbulence near the middle of the path. Considering for example a constant $C_{n}^{2}$ and no central obscuration $(q=0)$, we find $\mu_{F R} \approx$ 0.12 for $D_{F} / \sqrt{\lambda L} \rightarrow \infty$.

We now consider a link between space and ground, where the downlink is assumed to be the forward link. Since the atmosphere is much closer to the forward receiver than to the forward transmitter, we have $z_{2} \ll z_{1}$ and $D_{R}\left(z_{2} / z_{1}\right) \ll D_{F}$. Thus, Eq. (18) becomes

$$
H_{F R}\left(r ; z_{1}\right) \approx a_{R}\left(z_{2} / z_{1}\right)^{2} W_{F}(r) .
$$

In addition, for the return link (uplink), the correlation width at the receiver is much larger than the receiver size $\left(D_{R} \ll \rho_{R}\right)$, so that we can write

$$
2 \pi \int_{0}^{\infty} B_{R}(r) H_{R}(r) r \mathrm{~d} r \approx a_{R}^{2} B_{R}(0)
$$

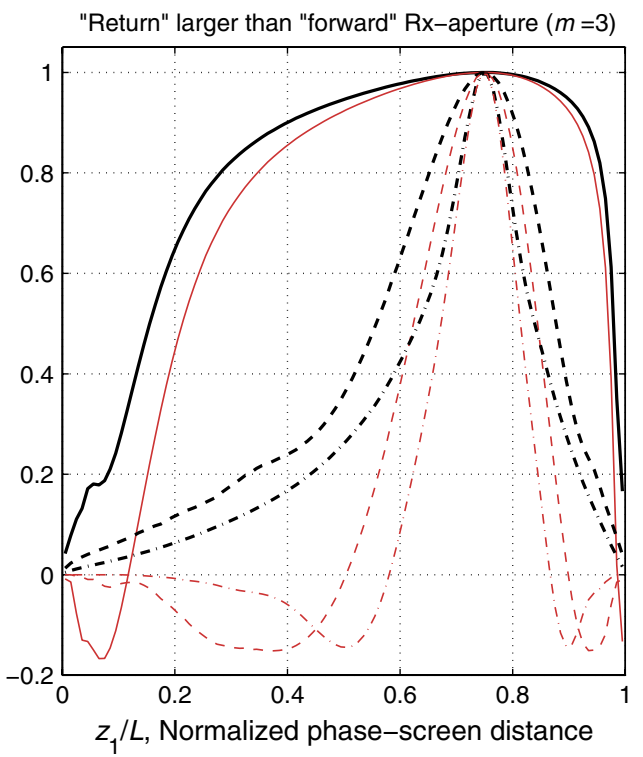

Fig. 2. (Color online) Correlation coefficient $\mu_{F R}$ between forward and return Rx powers for the case of a single turbulent screen along the path. Left: Rx apertures of the forward and return channels with the same geometry $(m=1)$. Right: Rx aperture of the return channel 3 times larger than the forward-channel $\mathrm{Rx}$ aperture $(m=3)$. The key and the $y$-axis are common to both graphs. Two values for the obscuration ratio $q$ (ratio of inner to outer diameter) are considered. 


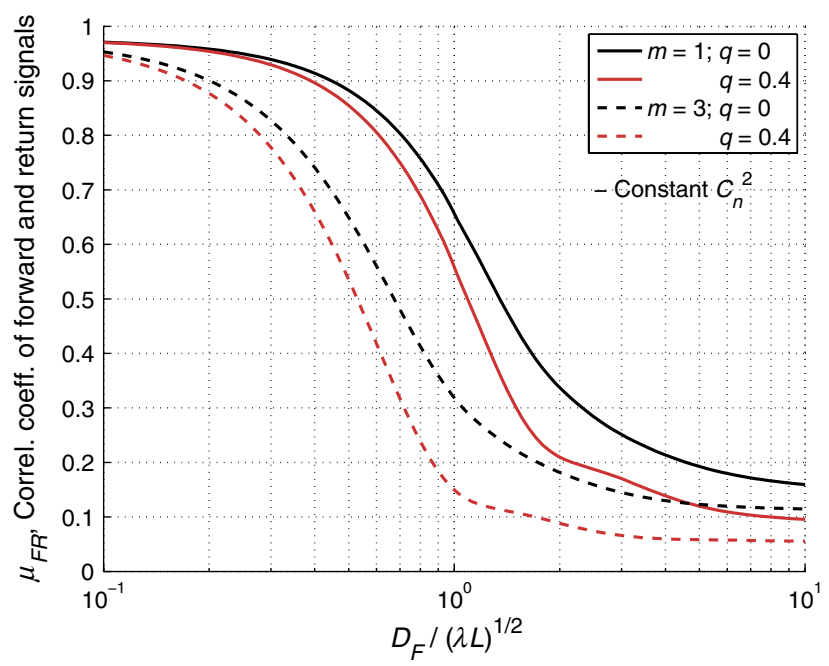

Fig. 3. (Color online) Correlation coefficient $\mu_{F R}$ as a function of the normalized Rx diameter for the case of a constant- $C_{n}^{2}$ path.

Finally, introducing Eqs. (23) and (24) into Eq. (22), we obtain

$$
\mu_{F R}=\frac{\int_{0}^{\infty} B_{F}(r) W_{F}(r) r \mathrm{~d} r}{\sqrt{(2 \pi)^{-1} B_{R}(0) \int_{0}^{\infty} B_{F}(r) H_{F}(r) r \mathrm{~d} r}} .
$$

An important assumption underlying our analysis is that both transceivers should share the same axis. In general, the relative velocity of the spacecraft requires the transmitters to point ahead of the direction of the received beam. Thus, Eq. (25) is based on a negligible point-ahead angle, meaning that the point-ahead angle should be much smaller than the isoplanatic angle [10].

Figure $\underline{4}$ shows the correlation coefficient $\mu_{F R}$ for a space-ground bidirectional link calculated from Eq. (25) with a $C_{n}^{2}$ profile determined by the Hufnagel-Valley model "HV $\mathrm{H}_{5-7}$ " [11]. The vertical path may be slanted as long as the link remains

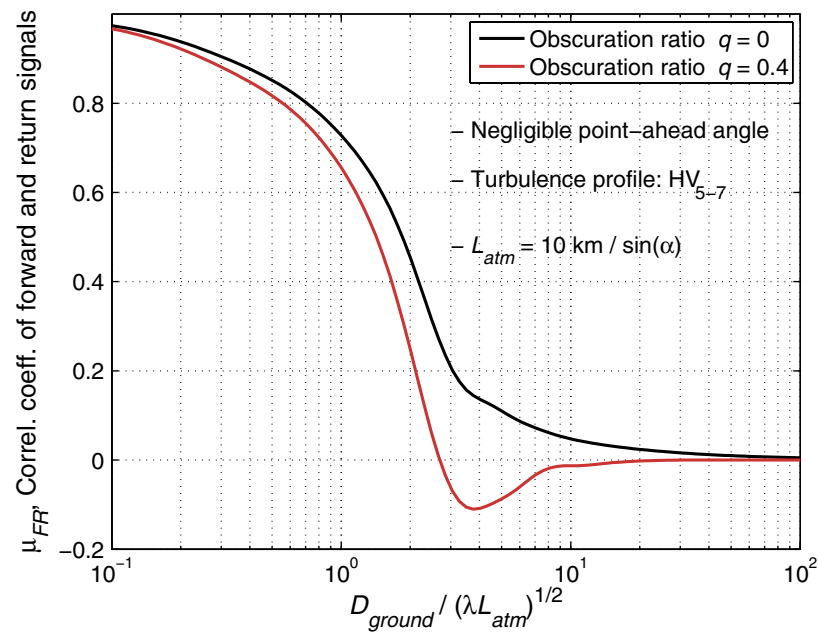

Fig. 4. (Color online) Correlation coefficient $\mu_{F R}$ as a function of the normalized Rx diameter on the ground for the case of one ground terminal and one on a spacecraft. in the weak-fluctuation regime and as long as Earth curvature has a negligible impact. The coefficient $\mu_{F R}$ is plotted versus $D_{\text {ground }} / \sqrt{\lambda L_{\text {atm }}}$, where $D_{\text {ground }}$ is the ground receiver's diameter and $L_{\text {atm }}$ is the characteristic atmospheric propagation length, which has been set to $10 \mathrm{~km} / \sin (\alpha), \alpha$ being the elevation angle of the link above the horizon. Following our reasoning in Section $\underline{4}$, the configuration that yields a strong effect on the central obscuration can be deduced from the relation $q D_{F} / \rho_{F}-D_{R} / \rho_{R} \approx 1$. Recalling that $D_{R} \ll$ $\rho_{R}$ and identifying $D_{F} / \rho_{F} \approx D_{\text {ground }} / \sqrt{\lambda L_{\text {atm }}}$, we conclude that the central obscuration significantly reduces the correlation at $D_{\text {ground }} / \sqrt{\lambda L_{\text {atm }}} \approx 1 / q$.

\section{Conclusion}

We have extended the point-source point-receiver (PSPR) scenario to a finite receiver that spatially averages scintillation over its aperture. For the sake of simplicity, the assumption of a point source and its associated spherical wave has been kept. The correlation coefficient of the received powers could be expressed analytically using the Rytov (weakfluctuation) theory. The main factors impacting the correlation are (1) the position of the turbulence along the path, (2) the aperture size with respect to the Fresnel length $\sqrt{\lambda L}$, and (3) the aperture's central obscuration.

The accuracy of these results may be improved by extending the analysis to the strong-scintillation regime. Although more complex, several approaches for the characterization of strong scintillation exist $[\underline{7,12}, \underline{13}]$ and might yield more general expressions of the correlation coefficient.

After having characterized the PSPR channel reciprocity, it would be interesting to assess its usefulness, in particular for free-space optical communications. Knowing the channel state (scintillation fade or surge), a transmitter can adapt the signal properties (e.g., power, data rate) accordingly and improve the channel capacity. As noted in [3], the channel reciprocity makes a dedicated channel-state feedback radio link unnecessary and allows a shortening of the channel-state feedback delay. The feedback delay is the difference between the time at which the feedback-dependent communication signal is perturbed by the channel and the time at which the channel was actually measured. This delay should be shorter than the scintillation correlation time (typically around $1 \mathrm{~ms}$ ). For the case of a conventional dedicated feedback link, the feedback delay equals the round-trip time, $2 L / c$ with $c$ the light speed, plus the processing time at both terminals. For the case of a feedback based on PSPR channel reciprocity, the delay depends on the position of turbulence along the path. Considering the simplified case depicted in Fig. 1 with a single PS located at a distance $z_{1}$ from the forward transmitter, the feedback delay of a forward link equals only $2 z_{1} / c$ plus the processing time at the forward transmitter. 
Future work could consist of evaluating the benefit of channel reciprocity, e.g., in decibels of average Tx power, for specific link scenarios. First, the dependence of the channel capacity on the reciprocity correlation must be determined so that a minimum correlation coefficient for a link performance increase can be identified. Then, an extensive link budget analysis must be performed considering that reciprocity correlation is reached at the expense of antenna gains and aperture averaging. In fact, we can already state that the need for small-aperture antennas to obtain significant correlation will make difficult the application of PSPR reciprocity to long-distance links. Links between a ground station and a spacecraft generally involve large apertures on the ground and also a point-ahead angle that may significantly reduce the correlation. For example, to maintain the sphericalwave assumption for an uplink, the beam diameter $D_{\text {Tx,ground }}$ at the transmitter should fulfill the condition $D_{\text {Tx ground }} \ll \sqrt{\lambda L_{\text {atm }}}$, where $L_{\text {atm }}$ is the characteristic atmospheric length. With $\lambda=1 \mu \mathrm{m}$ and $L_{\text {atm }}=10 \mathrm{~km}$, the condition becomes $D_{\text {Tx.ground }} \ll$ $100 \mathrm{~mm}$ and limits the applications to near-Earth spacecraft and low-bandwidth measurements.

\section{References}

1. J. H. Shapiro, "Reciprocity of the turbulent atmosphere," J. Opt. Soc. Am. 61, 492-495 (1971).

2. R. F. Lutomirski and H. T. Yura, "Propagation of a finite optical beam in an inhomogeneous medium," Appl. Opt. 10, 1652-1658 (1971).
3. D. Giggenbach, W. Cowley, K. Grant, and N. Perlot, "Experimental verification of the limits of optical channel intensity reciprocity," Appl. Opt. (to be published).

4. R. R. Parenti, J. M. Roth, J. Shapiro, and F. G. Walther "Observations of channel reciprocity in optical free-space communications experiments" in Applications of Lasers for Sensing and Free Space Communications, OSA Technical Digest (CD) (Optical Society of America, 2011), paper LTuD3.

5. V. P. Aksenov, V. A. Banakh, V. M. Buldakov, V. L. Mironov, and O. V. Tikhomirova, "Distribution of fluctuations of light intensity behind the objective of a telescope after reflection in a turbulent atmosphere," Sov. J. Quantum Electron. 15, 1404-1406 (1985).

6. V. A. Banakh and V. L. Mironov, LIDAR in a Turbulent Atmosphere (Artech House, 1987).

7. L. Andrews and R. Phillips, Laser Beam Propagation through Random Media 2nd ed. (SPIE, 2005).

8. J. Goodman, Introduction to Fourier Optics 3rd ed. (Roberts, 2005).

9. D. L. Fried, "Optical heterodyne detection of an atmospherically distorted signal wave front," Proc. IEEE, 55, 57-77 (1967).

10. S. Basu and D. Voelz, "Tracking in a ground-to-satellite optical link: effects due to lead-ahead and aperture mismatch, including temporal tracking response," J. Opt. Soc. Am. A 25 1594-1608 (2008).

11. J. W. Hardy, Adaptive Optics for Astronomical Telescopes (Oxford, 1998).

12. V. A. Banakh and A. Z. Vagner, "Calculation of the variance of the strong intensity fluctuations for light beams propagating in the turbulent atmosphere," Atmos. Oceanic Opt. 5, 24-28 (1992).

13. L. C. Andrews and R. L. Phillips, "Monostatic lidar in weakto-strong turbulence," Waves Random Media, 11, 233-245 (2001). 